\title{
diffusion-îundarrentals
}

The Open-Access Journal for the Basic Principles of Diffusion Theory, Experiment and Application

\section{Diffusion Measurements in Fluids by Dynamic Light Scattering}

\author{
Andreas P. Fröba and Alfred Leipertz \\ Lehrstuhl für Technische Thermodynamik (LTT), Universität Erlangen-Nürnberg, \\ Am Weichselgarten 8, D-91058 Erlangen, Germany, \\ E-Mail: sek@1tt.uni-erlangen.de
}

\begin{abstract}
In the course of the last thirty years, light scattering techniques have been used with increasing effort and attention for the measurement of the thermophysical properties of pure fluids and fluid mixtures. Here, an introduction is given to dynamic light scattering (DLS) as a valuable tool for the measurement of diffusion processes. First, the basic principles of the method and its experimental realization are presented in some detail. A survey on various applications is given, which especially are related to the determination of transport and other thermophysical properties of transparent fluids. Selected measurements and results are shown for the determination of the thermal diffusivity $a$ in pure fluids and fluid mixtures. For the latter the experimental conditions can turn out to be more complex. The measurement of the mutual diffusion coefficient $D_{12}$ in binary fluid mixtures, however, is also treated with the simultaneous determination of $a$ which can be realized under certain conditions. In this context beside results for fluids of technical interest, e.g., alternative refrigerants, typical measurements within the critical region for a binary fluid mixture along the critical isochor and a critical separation system are highlighted. Beside $a$ and $D_{12}$ the application of light scattering from bulk fluids on a molecular level gives also information about sound speed $c_{\mathrm{S}}$, sound attenuation $D_{\mathrm{S}}$, and Landau-Placzek ratio $S$. For these properties some results from DLS are renewed for the hydrofluorocarbons 1,1,1,2-tetrafluoroethane and pentafluoroethane. Additionally, the measurement of the dynamic viscosity $\eta$ based on the determination of the diffusion coefficient $D_{\mathrm{P}}$ of suspended seed particles is demonstrated for selected pure fluids. The application of DLS to a liquid-vapor interface, also called surface light scattering (SLS), for the simultaneous determination of liquid kinematic viscosity $v$ and surface tension $\sigma$ is demonstrated for the important and, thus, well-documented reference fluid toluene, pure refrigerants and their mixtures, and a high viscosity fluid. These measurements demonstrate an excellent performance of the SLS-technique regarding both the achievable high accuracy and the application over a wide range of viscosity.
\end{abstract}

KEY WORDS: dynamic light scattering; dynamic viscosity; kinematic viscosity; mutual diffusion coefficient; particle diffusion coefficient; sound attenuation; sound speed; surface light scattering; surface tension; thermal diffusivity 


\section{Introduction}

Diffusion measurements in fluids mainly stand for the accurate determination of transport properties. Important quantities are the thermal diffusivity $a$, the mutual diffusion coefficient $D_{12}$, the sound attenuation $D_{\mathrm{S}}$, the particle diffusion coefficient $D_{\mathrm{P}}$, and the dynamic viscosity $\eta$ or kinematic viscosity $v$. A fundamental problem which arises with the conventional determination of these quantities is that a gradient has to be applied which must be large enough to give rise to a measurable effect and small enough to cause only very little perturbation in the system under investigation. This difficulty is strongly enhanced in the vicinity of critical points, where induced gradients may make any reasonable measurement impossible. However, information collected in these regions is of great importance both for a fundamental understanding of critical phenomena and for setting up correlations for the individual properties of a substance.

A very successful approach to overcome these limitations is the application of light scattering techniques [1-4]. A fundamental advantage of these methods is given by the fact that these may be used in thermodynamic equilibrium. They make use of microscopic statistical fluctuations, which may be related to various diffusive processes and thus to the transport properties to be measured. The basic justification for this relation is Onsager's regression hypothesis [5], which states that, on a statistical average, microscopic fluctuations are governed by the very same macroscopic transport equations and which has been confirmed also by light scattering investigations.

\section{Basic Principles}

In the following, the underlying theory of dynamic light scattering (DLS) from bulk fluids and the application of this method to fluid surfaces also called surface light scattering (SLS) is briefly summarized. For a more detailed and comprehensive description the reader is referred to the specialized literature [6-9].

\subsection{Spectra of Scattered Light}

\subsubsection{Light Scattering from Bulk Fluids}

When a fluid sample in macroscopic thermodynamic equilibrium is irradiated by coherent laser light, light scattered from the sample can be observed in all directions. The underlying scattering process is governed by microscopic fluctuations of temperature (or entropy), of pressure, and of species concentration in mixtures. The relaxation of these statistical fluctuations follow the same laws which are valid for the relaxation of macroscopic systems. Thus, the decay of temperature fluctuations is governed by the thermal diffusivity $a$. Pressure fluctuations in fluids are moving with sound speed $c_{\mathrm{S}}$ and their decay is governed by the sound attenuation $D_{\mathrm{S}}$. In the case of a binary fluid mixture, the decay of fluctuations in concentration is governed by the mutual diffusion coefficient $D_{12}$.

A typical scattering geometry for light scattering from bulk fluids is shown in Fig. 1. With the scattering angle $\Theta_{\mathrm{S}}$, which defines the angle between the direction of 


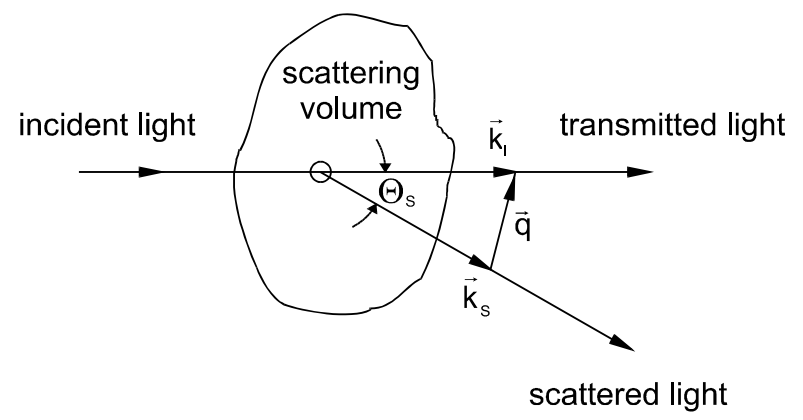

Figure 1 Scattering geometry.

observation and incident light, the scattering vector is determined by $\vec{q}=\vec{k}_{\mathrm{I}}-\vec{k}_{\mathrm{S}}$. Here, the wave vectors of incident and scattered light are represented by $\vec{k}_{\mathrm{I}}$ and $\vec{k}_{\mathrm{S}}$, respectively. Assuming elastic scattering ( $k_{\mathrm{I}} \cong k_{\mathrm{S}}$ ), the modulus of the scattering vector is given by the fluid refractive index $n$, the laser wavelength in vacuo $\lambda_{0}$, and the scattering angle $\Theta_{\mathrm{S}}$ by $[9]$

$$
q=\left|\vec{k}_{\mathrm{I}}-\vec{k}_{\mathrm{S}}\right| \cong 2 k_{\mathrm{I}} \sin \left(\Theta_{\mathrm{S}} / 2\right)=\frac{4 \pi n}{\lambda_{0}} \sin \left(\Theta_{\mathrm{S}} / 2\right) .
$$

The above-mentioned equalization processes result in a characteristic spectrum of the scattered light [6], which is shown schematically in Fig. 2. The temperature and/or concentration fluctuations contribute to the central or unshifted Rayleigh component of

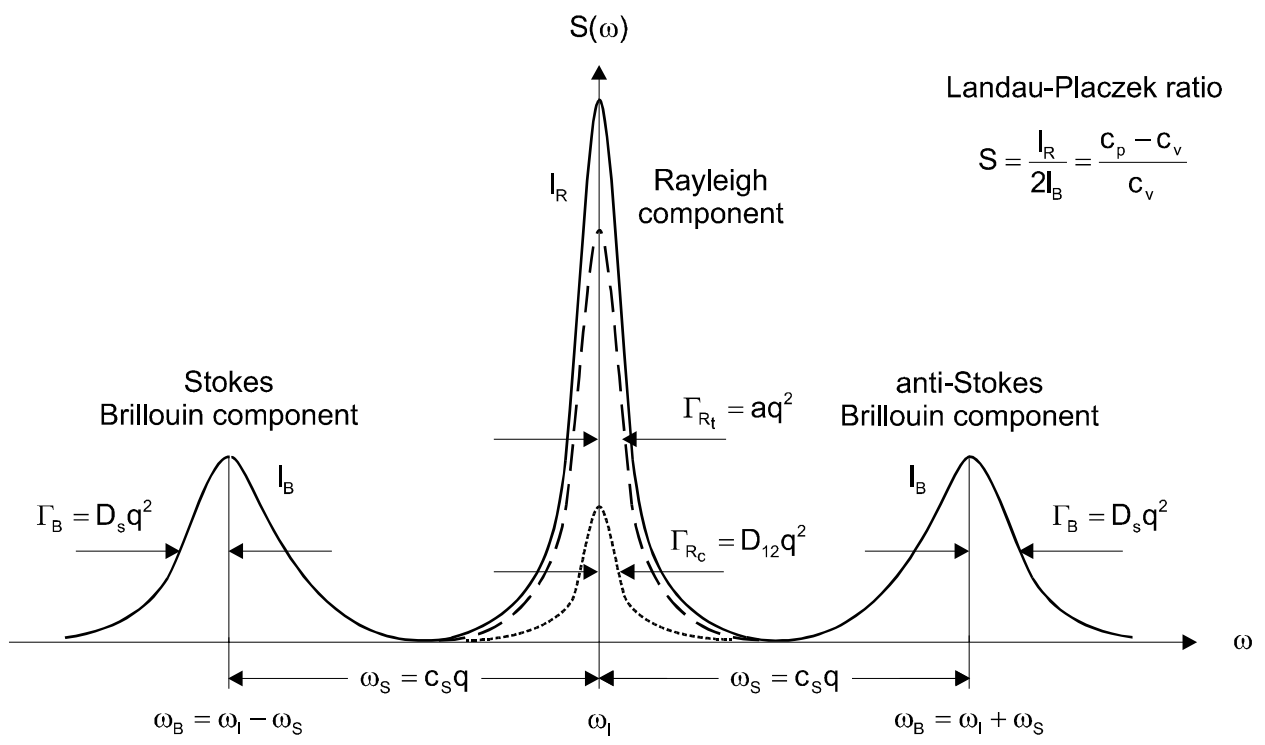

Figure 2 Schematic representation of the spectrum of scattered light for a binary fluid mixture. 
the spectrum of the scattered light. The pressure fluctuations are responsible for the Brillouin lines shifted relative to the frequency $\omega_{\mathrm{I}}$ of the incident light by $\omega_{\mathrm{S}}$. The widths of these three lines, which in good approximation all exhibit a Lorentzian form, yield information on the relaxation of fluctuations in the fluid and thus on the values of the transport coefficients and other thermophysical properties. In particular, the widths of the Brillouin lines are governed by the sound attenuation $D_{\mathrm{S}}$, the width of the Rayleigh line is determined by the thermal diffusivity $a$, and, in case of a binary fluid mixture, also by the mutual diffusion coefficient $D_{12}$. The frequency spacing $\omega_{\mathrm{S}}$ between the Rayleigh and Brillouin lines is proportional to the speed of sound $c_{\mathrm{S}}$. Additionally, the signal ratio of the Rayleigh to Brillouin lines, the Landau-Placzek ratio $S=I_{\mathrm{R}} / 2 I_{\mathrm{B}}$, contains information on the specific heats, $c_{\mathrm{p}}$ and $c_{\mathrm{v}}$, at constant pressure and volume, respectively.

When adding seed particles to the fluid, the spectrum of scattered light is normally dominated by the particle scattering process resulting in a frequency unshifted line. For monodisperse spherical particles the line exhibits a pure Lorentzian shape and its width, $\Gamma_{\mathrm{P}}=D_{\mathrm{P}} q^{2}$, is governed by the tanslational particle diffusion coefficient $D_{\mathrm{P}}$, which is related via the Stokes-Einstein equation, $D_{\mathrm{P}}=k_{\mathrm{B}} T /(3 \pi \eta d)$, with dynamic viscosity $\eta$, particle diameter $d$, Boltzmann's constant $k_{\mathrm{B}}$, and temperature $T[10,11]$. Thus, several different thermophysical properties of interest can be found nearly simultaneously by analysing the spectrum of the scattered light.

\subsubsection{Light Scattering by Surface Waves}

Surface light scattering (SLS) is a technique which is closely related to DLS in its classical meaning. The difference is that this technique probes, as the name indicates, fluctuations on the surface of a liquid or, in a more general formulation, at phase boundaries. These fluctuations can be understood as thermally excited capillary waves. Here, for an introduction to the technique a more simplified view is given, which, e.g., neglects the presence of a second fluid phase and thus refers to capillary waves on a free liquid surface. For an exact treatment of the capillary wave problem, necessary for the determination of viscosity and surface tension with high accuracy, the reader is referred to literature [12-14].

In macroscopic thermal equilibrium, liquid surfaces exhibit surface waves that are caused by the thermal motion of molecules and that are quantized in so-called "ripplons" [15]. The existence of fluctuations driven by the thermal motion constantly distorts the flat equilibrium state of a surface, and the surface should be rough on average which was first predicted by Smoluchowski in 1908 [16]. A thermally excited surface can be represented by a sum of Fourier components which means a superposition of surface waves with different amplitudes $\xi_{q}$ and wave vectors $\vec{q}$ [8]; see Fig. 3. Light interacting with an oscillating surface structure is scattered. Each Fourier component of the rough surface behaves optically as a weak phase grating and scatters a small fraction of the incident light in equally spaced directions around both the reflected and refracted beams. Thermally excited surface fluctuations observable in the light scattering experiment cover 


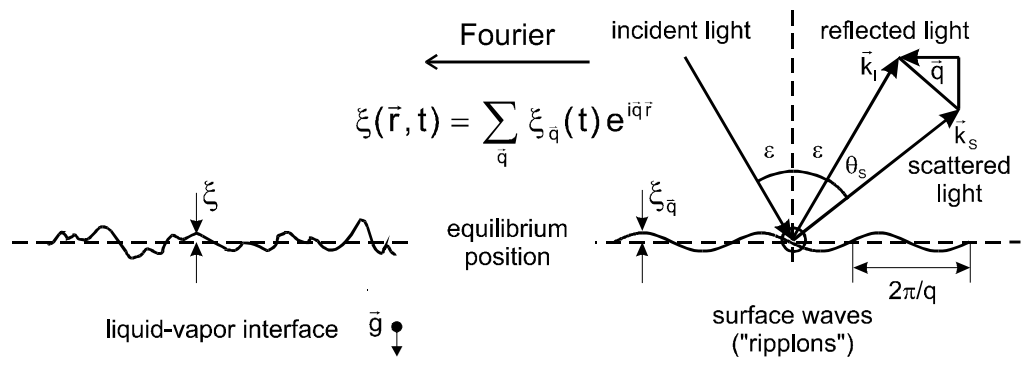

Figure 3 Representation of a liquid-vapor interface by a superposition of surface waves with different amplitudes and wavelength.

a typical range of wavelengths from about 0.1 to $1000 \mu \mathrm{m}$; see, e.g., Refs. 8, 17-19. The total root-mean-square amplitude of the surface roughness integrated over all wavelengths, where a lower limit of a few molecular sizes is assumed, is typically between 1 and $100 \mathrm{~nm}$ [8]. Of course, the amplitude of a given Fourier component is much smaller.

In order to excite surface fluctuations, work has to be done against the forces acting on a liquid surface. Due to the typically small values of the wavelengths and amplitudes, capillary forces dominate, while gravitational forces can be neglected. For this reason the temporal evolution of surface or capillary waves is governed by surface tension and by surface and bulk viscoelasticity. Depending on the reduced capillary number $Y=\sigma \rho /\left(4 \eta^{2} q\right)$, for the temporal decay of surface fluctuations, two cases may be distinguished. In the case of large viscosity and/or small surface tension when $Y \leq 0.145$, the amplitude of surface waves is damped exponentially, while in the case of small viscosity and/or large surface tension when $Y>0.145$, the amplitude decays in the form of a damped oscillation.

The scattering geometry typically used for light scattering experiments on liquid surfaces is shown in Fig. 3, where scattered light is observed near reflection. By choice of the angle of incidence $\varepsilon$ and the scattering angle $\Theta_{\mathrm{S}}$, the scattering vector $\vec{q}=\vec{k}_{\mathrm{I}}^{\prime}-\vec{k}_{\mathrm{S}}^{\prime}$ is determined and, from this, the wave vector and frequency of the observed surface vibration mode. Here, $\vec{k}_{\mathrm{I}}^{\prime}$ and $\vec{k}_{\mathrm{S}}^{\prime}$ denote the projections of the wave vectors of the reflected $\left(\vec{k}_{\mathrm{I}}\right)$ and scattered light $\left(\vec{k}_{\mathrm{S}}\right)$ in the surface plane, respectively. For the observation of scattered light within the irradiation plane and assuming elastic scattering (i.e., $k_{\mathrm{I}} \cong k_{\mathrm{S}}$ ), the modulus of the scattering vector is given by $[14,20]$

$$
q=\left|\vec{k}_{\mathrm{I}}^{\prime}-\vec{k}_{\mathrm{S}}^{\prime}\right| \cong 2 k_{\mathrm{I}} \sin \left(\Theta_{\mathrm{S}} / 2\right) \cos \left(\varepsilon-\Theta_{\mathrm{S}} / 2\right)=\frac{4 \pi n}{\lambda_{0}} \sin \left(\Theta_{\mathrm{S}} / 2\right) \cos \left(\varepsilon-\Theta_{\mathrm{S}} / 2\right),
$$

where $n$ is the fluid refractive index and $\lambda_{0}$ is the laser wavelength in vacuo.

The optical or first-order power spectrum of the scattered electric field at a point in the far field reflects ideally the power spectrum of a particular surface mode. An exponential decay of surface waves results solely in a broadening of the spectrum, 


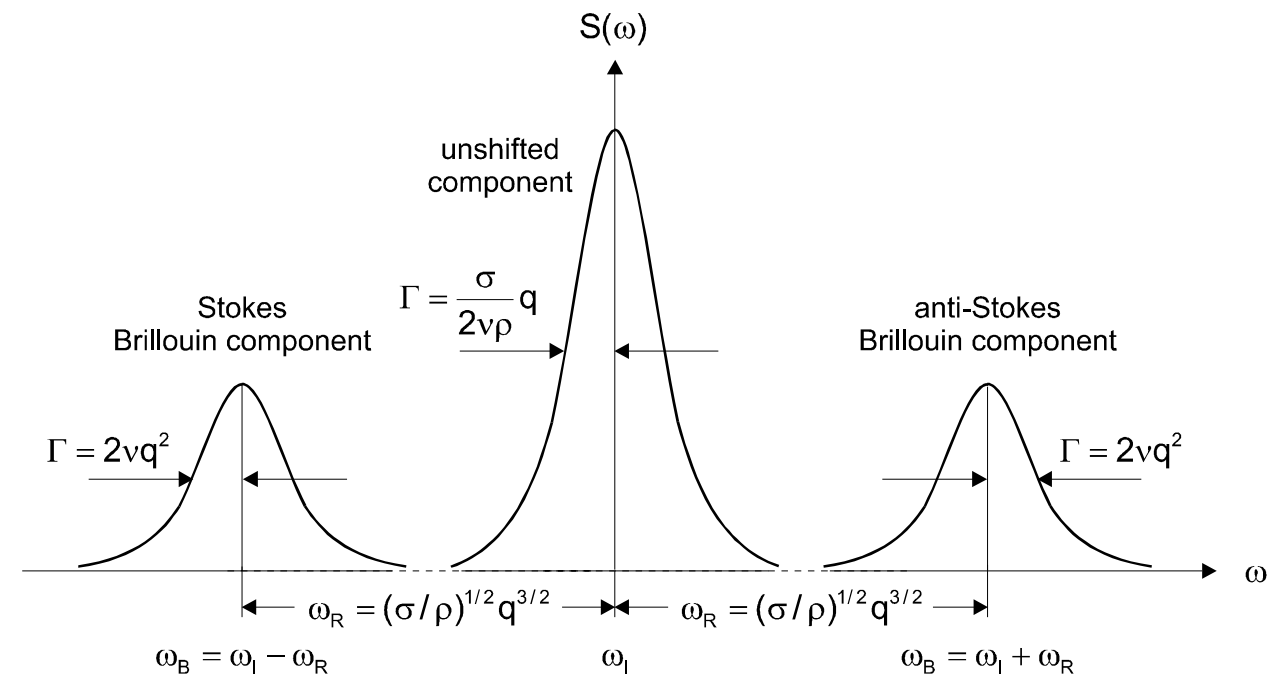

Figure 4 Spectrum of scattered light by surface waves: frequency unshifted line (center) in the case of large viscosity and/or small surface tension $(Y<<0.145)$; frequency shifted Brillouin lines in the case of small viscosity and/or large surface tension $(Y>0.145)$.

whereas an oscillatory damping gives rise to a Brillouin-doublet. For a liquid surface, line widths and separation are related to the modulus of the scattering vector $q$, surface tension $\sigma$, density $\rho$, and kinematic viscosity $v$ as shown in Fig. 4 in a first-order approximation [8]. A Lorentzian shape of these lines, as well as the relations for line widths and separations as indicated in Fig. 4, only hold in the limiting cases $Y>0.145$ or $Y<<0.145$, respectively. For intermediate values of $Y$ the line shapes become more complex. Here, apart from the limiting case of $Y$ values close to the critical value of 0.145 , the spectrum is found to be identical to the spectrum of the coordinate of a thermally excited harmonic oscillator [21].

\subsection{Correlation Technique}

The use of classical interference spectroscopy (Fabry-Perot spectroscopy), where one first spectrally filters the scattered light and then measures the intensity at a given frequency $\omega$ transmitted by the filter, seems to be the straightforward way to analyse the spectra of scattered light. Yet, this pre-detection filtering scheme is only possible under special conditions, for instance for light scattering from bulk fluids in the transition region from the kinetic to the hydrodynamic regime where the Rayleigh line is relatively broad [22, 23]. Usually, the width of the Rayleigh lines of order MHz or below for most cases of practical interest is such small that it is far beyond the resolving power of FabryPerot interferometers. This is the reason for analyzing the spectrum of the scattered light in a post-detection filtering scheme, where the total intensity is first detected and the 
detector signal is later filtered and processed. In this type of detection one measures optional the second-order power spectrum of the scattered light or, as in the following is described in some detail, the time-dependent intensity correlation function which also is named second-order correlation function

$$
G^{(2)}(\tau) \equiv\langle I(0) I(\tau)\rangle=\lim _{T \rightarrow \infty} \frac{1}{2 T} \int_{-T}^{T} I(t) I(t+\tau) d t .
$$

The brackets indicate the time average of the product $I(t) I(t+\tau)$ of the time-dependent intensity at two times $t$ and $t+\tau$. The spectral range has an upper limit of about $20 \mathrm{MHz}$, corresponding to the time resolution of the correlator instrument.

In a realistic experimental situation besides the light scattered from the fluctuations in a sample there may also be a contribution from stray light, e.g., from dust on the cell windows or from the cell windows themselves. Therefore, for a general description, we consider a local oscillator field coherent with the incident electric field. In terms of the total electric field $E(t)=E_{\mathrm{S}}(t)+E_{\mathrm{LO}}(t)$, which represents a superposition of the scattered electric field $E_{\mathrm{S}}(t)$ with the local oscillator field $E_{\mathrm{LO}}(t)$, the intensity or, more precisely, the radiative flux,

$$
I(t)=\frac{c \varepsilon}{2} E^{*}(t) E(t)
$$

measured by a detector is given by

$$
I(t)=\frac{c \varepsilon}{2}\left|E_{\mathrm{S}}(t)+E_{\mathrm{LO}}(t)\right|^{2}=I_{\mathrm{S}}(t)+I_{\mathrm{LO}}(t)+\frac{c \varepsilon}{2}\left[E_{\mathrm{S}}^{*}(t) E_{\mathrm{LO}}(t)+E_{\mathrm{LO}}^{*}(t) E_{\mathrm{S}}(t)\right],
$$

where the asterisk denotes the complex conjugate variable, $c$ the speed of light, and $\varepsilon$ the permittivity of the medium. $E_{\mathrm{S}}(t)$ and $E_{\mathrm{LO}}(t)$ can be specified more precisely with the help of the electric field of the incident light and the characteristic time behavior of the fluctuations observed [24]. By using some mathematical manipulations and fundamental relations it appears that the intensity correlation function accessible in light scattering experiments gives access to the correlation function of the fluctuations observed. For Eq. (3) one obtains

$$
G^{(2)}(\tau)=\left(I_{\mathrm{S}}+I_{\mathrm{LO}}\right)^{2}+I_{\mathrm{S}}^{2}\left|g_{\mathrm{q}}(\tau)\right|^{2}+2 I_{\mathrm{S}} I_{\mathrm{LO}} g_{\mathrm{q}}(\tau)
$$

where $I_{\mathrm{S}}, I_{\mathrm{LO}}$, and $g_{q}(\tau)$ are the time average of the intensity of the scattered light, the time average of the intensity of the local oscillator, and the normalized correlation function of the fluctuations observed. In deriving Eq. (6) it has been assumed that only one individual component dominates the spectrum of scattered light. This is equivalent to say that only fluctuations in one property of the medium cause fluctuations in the scattered light intensity.

\subsubsection{Light Scattering from Bulk Fluids}

In the case of a fluid in the hydrodynamic regime where the mean free path $r_{0}$ of the molecules is much smaller than the reciprocal value of the modulus of the scattering vector $q\left(q r_{0}<<1\right)$ and by this Onsager regression hypothesis holds, the time correlation functions of the fluctuations in the thermophysical properties of state can be derived on 
the basis of classical hydrodynamics. Because the fluctuations around the equilibrium values of temperature (or entropy), pressure, and in addition of species concentration in fluid mixtures are expected to be very small, the set of the linearized equations of fluid mechanics can be used. For the hydrodynamic modes of a fluid the correlation functions $g_{q}(\tau)$ can be found ultimately from the linearized hydrodynamic equations by applying a Fourier-Laplace analysis [6]. As for many other non-periodic statistical processes, such as for the diffusion of particles in dispersions, also for the statistical fluctuations of temperature (or entropy) and species concentration in a fluid mixture, the normalized time correlation function decays like a single exponential,

$$
g_{q}(\tau)=\exp \left(-\tau / \tau_{\mathrm{C}}\right),
$$

where $\tau_{\mathrm{C}}=\left(D q^{2}\right)^{-1}$ is called the "relaxation time" or the "correlation time" of the property, which reflects the mean decay behavior or life-time of the fluctuations observed. Here, $D$ may be identified to be the thermal diffusivity $a$, the mutual diffusion coefficient $D_{12}$ or the particle diffusion coefficient $D_{\mathrm{P}}$, respectively. For the local pressure fluctuations at constant entropy, which can be represented, to a good approximation, by propagating sound waves, the normalized time correlation function has the form

$$
g_{q}(\tau)=\cos \left(\omega_{\mathrm{S}} \tau\right) \exp \left(-\tau / \tau_{\mathrm{C}}\right)
$$

where the correlation time $\tau_{\mathrm{C}}=\left(D_{\mathrm{S}} q^{2}\right)^{-1}$ is related to the sound attenuation $D_{\mathrm{S}}$. In addition, the frequency $\omega_{\mathrm{S}}=c_{\mathrm{S}} q$, which is identical with the frequency of the sound waves observed, gives information about the sound velocity $c_{\mathrm{S}}$.

\subsubsection{Light Scattering by Surface Waves}

For the hydrodynamic fluctuations on a liquid surface the time correlation functions $g_{q}(\tau)$ can also be found ultimately from the solution of the linearized hydrodynamic equations expressing mass and momentum conservation [8]. Hereby the fluid flow must satisfy boundary conditions that express that normal and tangential stresses exerted on a small element of the interface are identical on the two sides of the surface. In addition, all fluid motion must vanish at an infinite distance from the interface. The theoretical approach, however, neglects the time of propagation and dissipation of the rotational flow in the bulk. This effect can be neglected, except in the region close to the critical damping $(Y \sim 1)$ where the dynamics of surface fluctuations may change between an oscillatory and an over-damped behavior. For this special case, a detailed theoretical treatment of the surface wave problem and its resolution can be found in Ref. 25 .

In the limiting case of small viscosity and/or large surface tension $(Y>>0.145)$ the normalized correlation function of surface fluctuations on a free liquid surface is given by

$$
g_{q}(\tau)=\cos \left(\omega_{\mathrm{R}} \tau\right) \exp \left(-\tau / \tau_{\mathrm{C}}\right),
$$

where the correlation time $\tau_{\mathrm{C}}=\left(2 v q^{2}\right)^{-1}$ and the frequency $\omega_{\mathrm{R}}=(\sigma / \rho)^{1 / 2} q^{3 / 2}$ are identical with the mean life time of "ripplons" and the frequency of propagation, respectively. In the limiting case of large viscosity and/or small surface tension $(Y<<0.145)$, the normalized correlation function of surface fluctuations on a free liquid surface is given by

$$
g_{q}(\tau)=\exp \left(-\tau / \tau_{\mathrm{C}}\right) .
$$


For this overdamped case the lifetime of "ripplons" is given by $\tau_{\mathrm{C}}=2 \eta / \sigma q$. For a reliable determination of viscosity and surface tension a more detailed and rigorous consideration of the surface light scattering method than given by Eq. (9) and (10) has to be applied. Here, the reader is referred to literature, see, e.g., Ref. 12-14.

Actually, even the correlation function $g_{q}(\tau)$ of a pure fluid does not consist of a simple exponential or damped oscillation, as the fluctuations in pressure and temperature are present simultaneously. The situation complicates, when fluid mixtures or particle dispersions are considered. However, as decay times and/or signal amplitudes are vastly different in many experimental situations, the ACF in a time interval of interest may often be analysed in terms of a simple exponential. This point will be described in more detail in the following sections.

\subsection{Homodyne and Heterodyne Techniques}

As can be seen from Eq. (6) in the presence of a local oscillator field the intensity correlation function consists of three terms, the constant term $\left(I_{\mathrm{S}}+I_{\mathrm{LO}}\right)^{2}$, the term $I_{\mathrm{S}}{ }^{2}\left|g_{\mathrm{q}}(\tau)\right|^{2}$, which is due to the scattered light alone and is denoted as homodyne term, and the heterodyne term $2 I_{\mathrm{S}} I_{\mathrm{LO}} g_{\mathrm{q}}(\tau)$. In the case that the correlation function of the hydrodynamic fluctuations $g_{\mathrm{q}}(\tau)$ decays like a single exponential we have the situation with two exponential functions with decay times different by a factor of two. As it is very difficult to extract reliable information from a sum of two exponentials with decay times of the same order of magnitude, the aim of the experimenter is to meet a situation where one of the exponentials clearly dominates. Either, one has to design the experiment in a way that only light from the sample itself is collected by the detector $I_{\mathrm{LO}}<<I_{\mathrm{S}}$. In this homodyne case, which may be the easier realized the larger the scattering cross section of the fluctuations is, the normalized intensity correlation function takes the form

$$
g^{(2)}(\tau)=1+\exp \left(-2 \tau / \tau_{\mathrm{C}}\right) .
$$

Alternatively, the strict heterodyne case is achieved. This will be done, if the signal is comparatively weak, and may be accomplished by deliberately adding part of the incident beam to the detected signal in a way that $I_{\mathrm{LO}} \gg I_{\mathrm{S}}$. Now the normalized intensity correlation function has the form

$$
g^{(2)}(\tau)=1+\frac{2 I_{\mathrm{S}}}{I_{\mathrm{LO}}} \exp \left(-\tau / \tau_{\mathrm{C}}\right) .
$$

It is crucial, however, in either case to make sure that one contribution clearly dominates. If not, there may be a considerable error, as the decay time determined from a fit to a supposed single exponential may be anywhere in between the two cases.

The derivation of Eq. (11) and (12), however, makes implicitly use of two idealizing assumptions, which in general do not hold. One simplification is that the scattered light is detected at a point in the far field. In practice, both the scattering volume and the area of detection are finite, which results in a deviation from an ideal coherent detection. Thus, there is an averaging effect with a reduction of the contrast $b=g^{(2)}(0)-1$ as compared to the value for a perfect registration. The deviation of $b$ from 1 or $2 I_{\mathrm{S}} / I_{\mathrm{LO}}$ in the case of a 
homodyne or heterodyne detection scheme, respectively, also takes into account effects of a finite speed of signal processing. Another simplification is the assumption of a constant intensity of the incident light. Slow fluctuations in the light source result in a deviation of the baseline from the ideal value 1 for the correlation function. Here, another adjustable parameter $a$ is introduced in practice. Thus, a practical ACF takes the form

$$
g^{(2)}(\tau)=a+b \exp \left(-2 \tau / \tau_{\mathrm{C}}\right)
$$

or

$$
g^{(2)}(\tau)=a+b \exp \left(-\tau / \tau_{\mathrm{C}}\right)
$$

assuming homodyne or heterodyne conditions, respectively.

The application of the heterodyne technique is especially useful for the evaluation of the periodic pressure fluctuations at constant entropy, which are responsible for the Brillouin components of the spectrum. In the case of a pure fluid a usual homodyne intensity ACF exhibits terms due to both pressure and temperature (or entropy) fluctuations and an additional cross term. To analyze the pressure fluctuations in a heterodyne detection scheme, the frequency of the local oscillator is shifted relative to the frequency $\omega_{\mathrm{I}}$ of the laser light by $\omega_{\mathrm{M}}$ applying an acousto-optical modulator. The frequency shift $\omega_{\mathrm{M}}$ is of the same order of magnitude as the frequency $\omega_{\mathrm{S}}$ of the pressure fluctuations observed $\left(\omega_{\mathrm{M}} \approx \omega_{\mathrm{S}}\right)$. By a proper choice of the intensity of the local oscillator shifted in frequency the signal governed by the periodic pressure fluctuations may be strongly enhanced as compared to that of the temperature (or entropy) fluctuations, and the correlation function takes the form of a damped oscillation

$$
g^{(2)}(\tau)=a+b \cos (\Delta \omega \tau) \exp \left(-\tau / \tau_{\mathrm{C}}\right) .
$$

Now the speed of sound $c_{\mathrm{S}}$ can be found from the knowledge of the adjusted modulator frequency $\omega_{\mathrm{M}}$ and the residual detuning $\Delta \omega=\left|\omega_{\mathrm{S}}-\omega_{\mathrm{M}}\right|$ of the correlation function according to $c_{\mathrm{S}}=\omega_{\mathrm{S}} / q=\left(\omega_{\mathrm{M}} \pm \Delta \omega\right) / q$. The sound attenuation $D_{\mathrm{S}}$ can be determined simultaneously from $\tau_{\mathrm{C}}$, see Sec.2.2.1.

Due to the usually small frequency separation of the shifted Brillouin-lines in the scattering spectrum for surface waves commonly no frequency shift of the coherent reference light is necessary.

\section{The Dynamic Light Scattering Experiment}

\subsection{Setup}

In this section only some information is given on the design of a light scattering apparatus. The exact choice of the individual components naturally depends strongly on the exact goal of the experiments, i.e., which property is to be measured. For details on the selection of the main components the reader is referred to literature [9]. A possible setup is displayed schematically in Fig. 5.

The main portion of the laser light is irradiated into a thermostated sample cell, beam splitters allow to add some reference light for a heterodyne detection, the frequency of which may be shifted for measurements on the Brillouin lines. Part of the scattered light is imaged, in the simplest case only by means of two circular stops, onto a detector, 


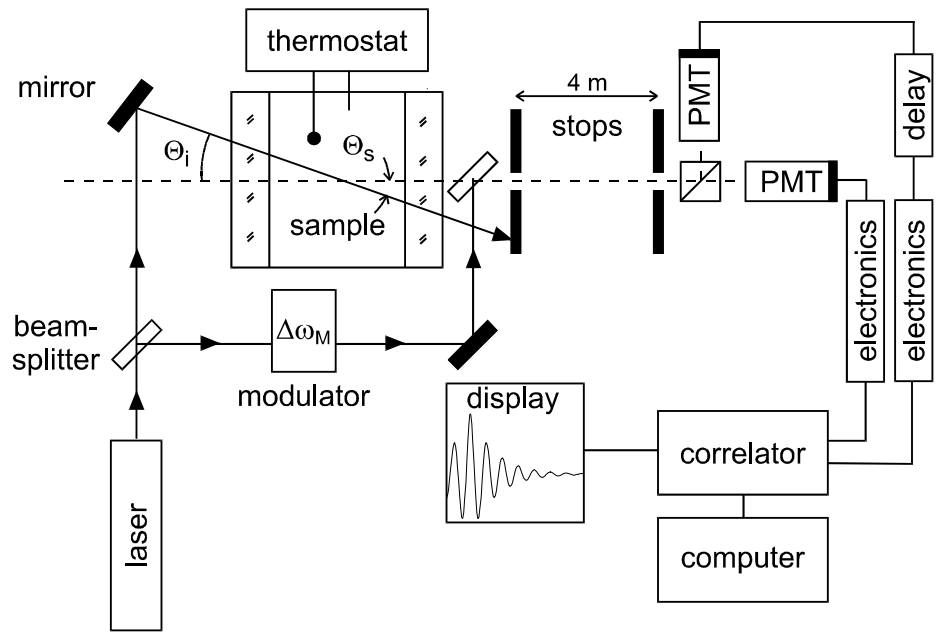

Figure 5 Possible setup for a dynamic light scattering experiment

conventionally a photo multiplier tube. The signal is amplified, discriminated and fed into the correlator, a special purpose computer for the computation of the ACF.

\subsection{Data Evaluation}

A central quantity for the evaluation of correlation functions is the decay time $\tau_{\mathrm{C}}$. In order to be able to extract this quantity with high accuracy, it is desirable to ensure experimental conditions where the ACF takes the form of a simple exponential. A curve of form $y=a+b \exp \left(-\tau / \tau_{\mathrm{C}}\right)$ is then fitted to the experimental ACF, which may be done using a non-linear algorithm according to Marquard and Levenberg [26]. In this context it is of great importance to make sure that the correlation function actually matches the theoretical model; if this does not hold there is a clear indication that the experiment cannot be relied on. A possible way to perform this check is to transform the experimental correlation function according to $g^{(2)}(\tau) \rightarrow \ln \left[g^{(2)}(\tau)-1\right]$ and to fit a polynom to this expression. In this method of cumulants [27] all orders higher than linear should vanish for a pure exponential and from the quadratic and the linear cofficient a quality factor may be built up to quantify deviations. However, there are two major problems connected with this approach. Firstly, it relies on a value of exactly one for the baseline; even small deviations, which may not affect the measurement in the time interval of interest, may result in erroneously bad values for the quality factor. Secondly, the transformation is basically restricted to positive values of $g^{(2)}(\tau)-1$; with experimental noise given, the cumulant expansion is restricted to a limited interval of lagtimes $\tau / \tau_{\mathrm{C}}$.

A different approach, which overcomes these problems is to fit an exponential to various lag time intervals [28]. The experimental ACF is only regarded to match the 

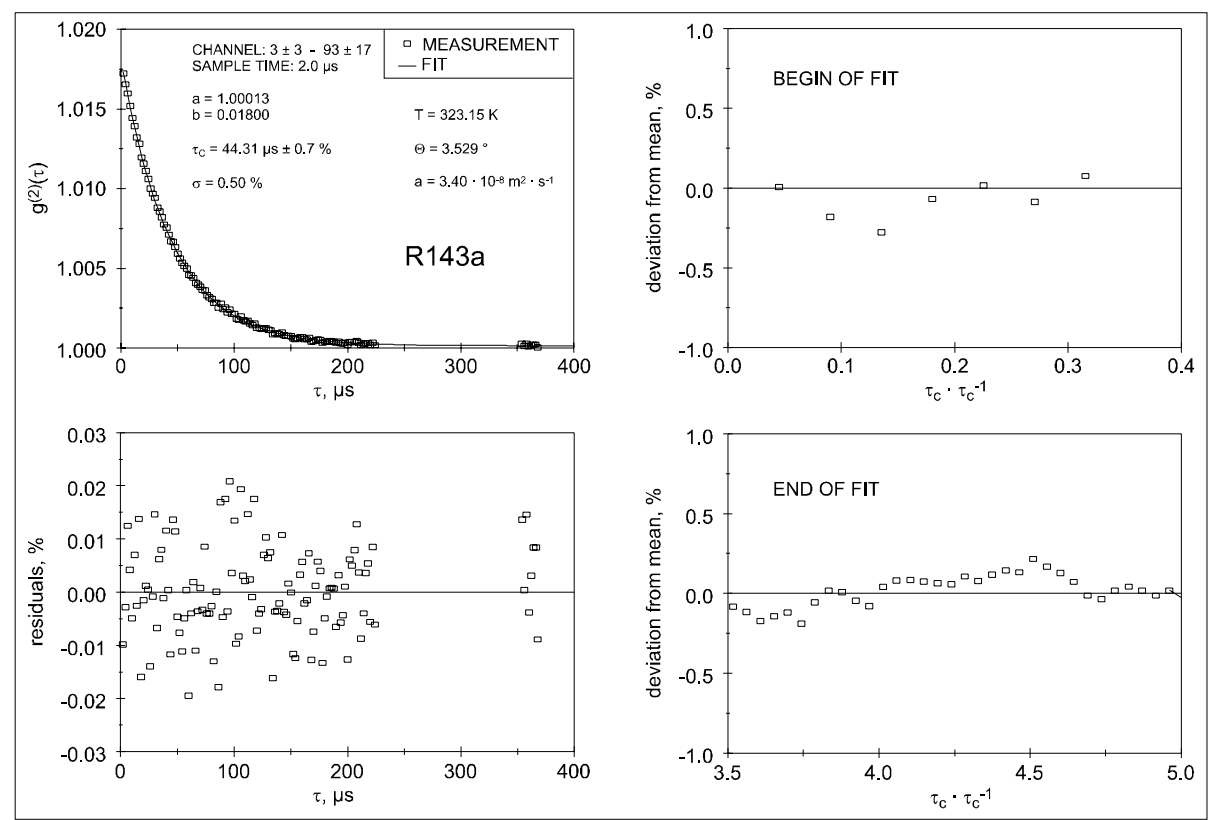

Figure 6 Example for the multi-fit approach for the evaluation of correlation functions. Insert on bottom left shows the residuals of experimental data and fit, which is free of systematic deviations. The insert on bottom right shows the deviation of the decay times determined from the mean value, when the fit is performed up to different lag times. The insert on top right shows the multi-fit procedure for the begin of the fit.

theoretical form if the decay times obtained for these different intervals agree within a certain value given. Moreover, the standard deviation of the single fit may be regarded as a measure of quality, which takes into account both the statistical quality of the experimental data and possible systematic deviations. An example for this procedure is given for a heterodyne detection scheme in Fig. 6, where a correlator with a single-tau structure has been used.

Another important question is that for the time interval that should be employed for the fit. If too many channels at large lag-times are omitted, there will be a significant loss of information; if the fit is extended too much into the background, noisy data without relevance are included. For the multi-fit procedure it has turned out to be advantageous if a span of about 2-6 decay times is regarded in the evaluation. This ensures that all relevant information is included and that possible deviations from a single exponential can be detected reliably. 


\section{Thermophysical Properties Measured by DLS}

\subsection{Thermal Diffusivity}

The thermal diffusivity $a$ is the transport property for which the development of the DLS technique is probably most advanced and where measurements can be carried out routinely over a wide range of temperature and pressure for the liquid phase and, due to the lower signal levels, in an extended vicinity of the critical point for the vapour phase [29-36]. What makes the measurement of the thermal diffusivity particularly interesting is the fact that it is hardly possible by any other than light scattering techniques to measure this property directly with a comparable accuracy. Other methods access the thermal conductivity $\lambda=a \rho c_{\mathrm{p}}$, which is related to $a$ by density $\rho$ and isobaric heat capacity $c_{\mathrm{p}}$.

With the exception of measurements in the vicinity of the critical point normally a heterodyne detection scheme is employed so that the correlation function takes the simple form of Eq. (12). As the measurement of the thermal diffusivity is performed at low scattering angles of about $2.5^{\circ}$ to $5^{\circ}$ an accurate measurement of the angle of incidence is of major importance. This may be performed by an autocollimation procedure with an uncertainty of order $0.01^{\circ}$ resulting in an uncertainty in the thermal diffusivity of about $0.5 \%$ to $1 \%$. The other major source of error is the determination of the decay time. If the correlation function is free from systematic error the uncertainty in the determination of $\tau_{\mathrm{C}}$ is given by the signal and the experimental duration. Depending on the equipment used, the scattering cross section of the fluctuations investigated and the time available for an experimental run, the total uncertainty for a single measurement is of order 1-3 \% [32]. If several independent experimental runs are performed, which is superior to running a single experiment over a longer time, this value may of course be improved.

There have been ample applications on the determination of $a$ for a wide range of fluids. DLS has especially contributed to an improvement in the data situation for new refrigerants [34-39]. An example is given in Fig. 7, where measurements on pure refrigerants and their mixtures with less environmental impact are shown.

Whereas the measurement of $a$ in pure fluids is basically a straightforward task, this is clearly more difficult in fluid mixtures. Even in the simplest case of a binary fluid mixture and neglecting the Brillouin component, the heterodyne correlation function takes the form of a sum of two exponentials

$$
g^{(2)}(\tau)=a+b_{1} \exp \left(-\tau / \tau_{\mathrm{C}, \mathrm{t}}\right)+b_{2} \exp \left(-\tau / \tau_{\mathrm{C}, \mathrm{c}}\right),
$$

where the subscripts $\mathrm{t}$ and $\mathrm{c}$ denote the contributions from temperature and concentration fluctuations, respectively. It is obvious that even for this simplified function the determination of the decay time $\tau_{\mathrm{c}, \mathrm{t}}$ is more complicated and associated with a higher degree of uncertainty than in the case of a pure fluid, because a larger number of parameters are to be fitted. The situation becomes easier if the refractive indices of the two components nearly match, as the signal from the concentration fluctuations may then be treated as a low-amplitude perturbation. A possible approach is to expand the 


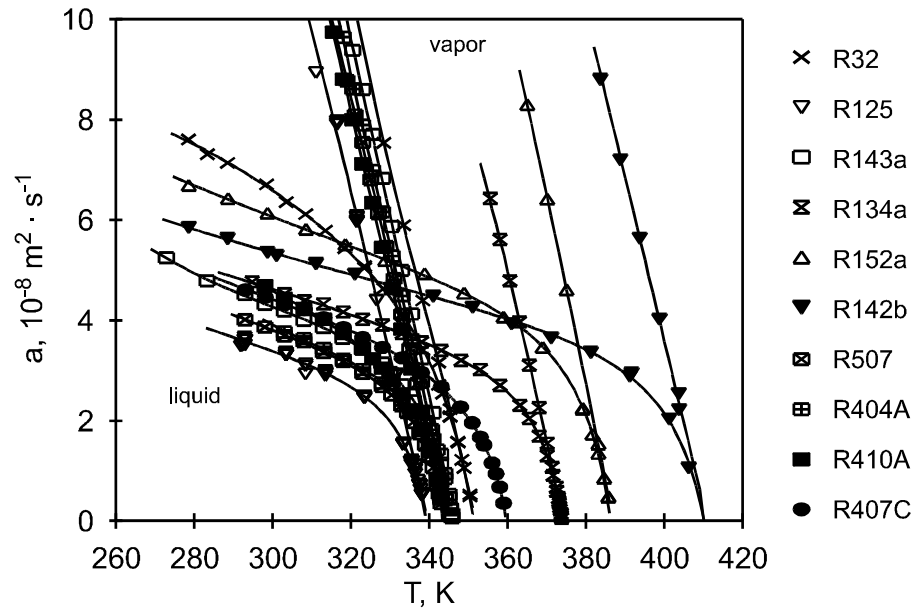

Figure 7 Thermal diffusivity of selected pure refrigerants and their mixtures on the saturation line [34, 37, 38]; upper symbols: liquid; lower symbols: vapor

exponential decay of the concentration fluctuations in a way that an ACF of form

$$
g^{(2)}(\tau)=a+b_{1} \exp \left(-\tau / \tau_{\mathrm{C}, \mathrm{t}}\right)+c \tau
$$

results [40]. The thermal diffusivity of a refrigerant mixture of R125 and R143a measured in that way is shown in Fig. 8. The experimental results were used for the validation of a prediction method which can be used in a straightforward way to obtain accurate information on different thermophysical properties of multicomponent mixtures over a wide temperature range in the two-phase region up to the critical point [39].

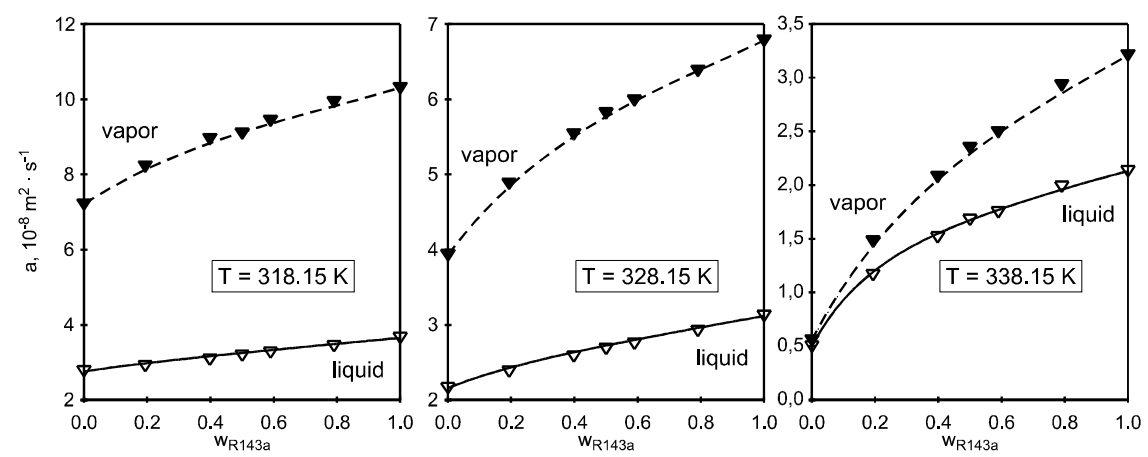

Figure 8 Thermal diffusivity of the binary mixture R-125/143a under saturation conditions and their dependence on the mass fraction of R143a at temperatures of 318.15 , 328.15 and $338.15 \mathrm{~K}$ [39]; symbols: DLS measurements; lines: prediction method. 
It is also possible to measure $a$ in mixtures with a larger difference in refractive index. Naturally, this is connected with a loss of accuracy and comes to a limit, when the signal due to temperature fluctuations is much weaker than that by concentration fluctuations.

\subsection{Mutual Diffusion Coefficient}

The problems encountered in measuring the mutual diffusion coefficient $D_{12}$ are of a similar nature as those of measuring the thermal diffusivity of mixtures. In either case there are basically signals from both temperature and concentration fluctuations. As the mutual diffusion coefficient is in general at least one order of magnitude smaller than the thermal diffusivity, larger angles may be chosen for the determination of this property. It is especially interesting, however, to perform the measurements with an identical setup as for $a$, as both properties may then be measured simultaneously. Whether one succeeds, is again governed by the actual separation of decay times and by the ratio of the signals.

As an example for a binary system with a large difference in the refractive indices, the thermal diffusivity and the mutual diffusion coefficient of an equimolar methane-ethane mixture are shown in Fig. 9 [41]. Measurements were performed over a wide temperature range between the plait critical point at $263.55 \mathrm{~K}$ and $310 \mathrm{~K}$ along the critical isochore. Due to the small signal amplitude of the fast mode, the thermal diffusivity could be obtained only with a relatively large measurement uncertainty. For the system methaneethane, however, the thermal diffusivity seems to remain finite when asymptotically approaching the plait critical point, while the mass diffusion coefficient reflects the critical slowing down of the order-parameter fluctuations and vanishes.

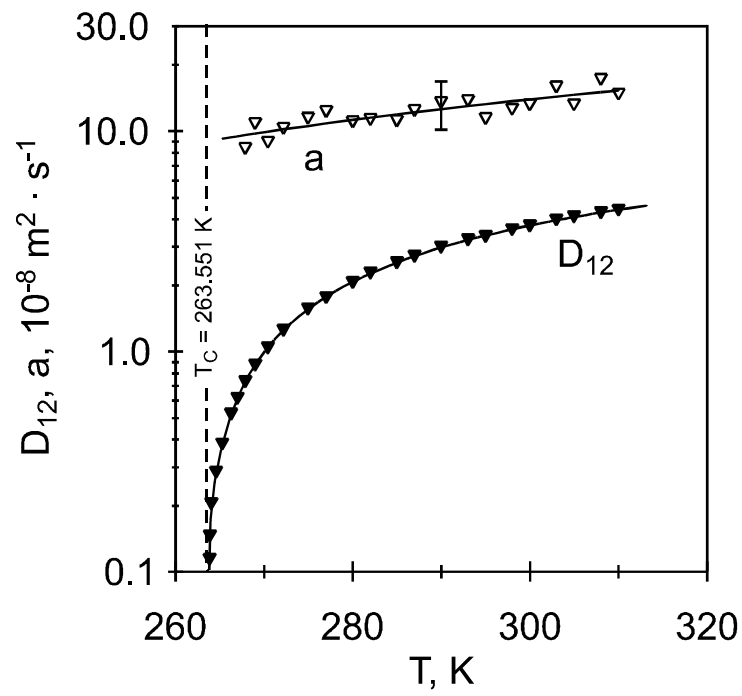

Figure 9 Thermal diffusivity and mutual diffusion coefficient of an equimolar methaneethane mixture along the critical isochor approaching the plait critical point [41]. 
In most instances, especially when the difference of refractive indices is large, the determination of the mutual diffusion coefficient may be performed by standard experiments. Fig. 10 shows the measured temperature dependence of the mutual diffusion coefficient of the separation system n-hexane / nitrobenzene [42]. According to the stability theory of thermodynamics, $D_{12}$ disappears at the critical separation point (temperature $T_{\mathrm{C}}$ ), which can be clearly seen from Fig. 10 .

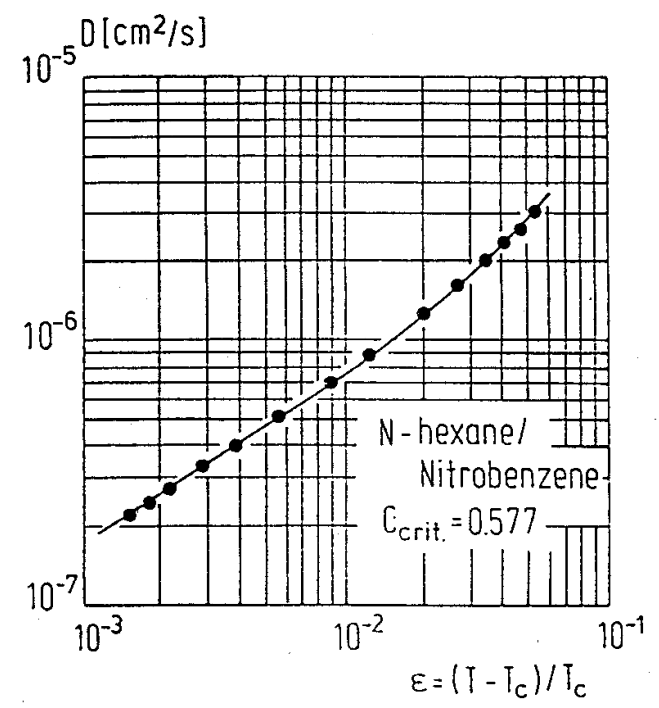

Figure 10 Mutual diffusion coefficient of a separation system as a function of the reduced temperature $\varepsilon=\left(T-T_{\mathrm{C}}\right) / T_{\mathrm{C}}[42]$.

\subsection{Sound Velocity and Sound Attenuation}

For the measurement of sound velocity $c_{\mathrm{S}}$ and sound attenuation $D_{\mathrm{S}}$ the Brillouin components of the spectrum are probed. As the Brillouin lines are shifted in frequency, a heterodyne technique with a local oscillator, which is also shifted in frequency by means of an acousto-optic modulator, is used [3]. This results in a correlation function in form of a damped oscillation, Eq. (15), where the sound attenuation is determined from the decay-time $\tau_{\mathrm{C}}$ as usual and the sound velocity is deduced from the known frequency shift by the modulator $\omega_{\mathrm{M}}$ and the residual mistuning $\Delta \omega$ of the correlogram. The simplest way of determining $\Delta \omega$ is by applying a Fourier transform to the measured correlation function, where the frequency is found from the maximum of the resulting spectrum [43]. DLS can be used to measure the sound velocity with a small uncertainty of about $0.5 \%$, and the technique has again been often applied to various refrigerants [34-39, 44]. An example is depicted in Fig. 11 [44].

In determining the sound attenuation one has to face two problems. One is that due to the large width of the Brillouin lines the decay-time of the correlation function is 


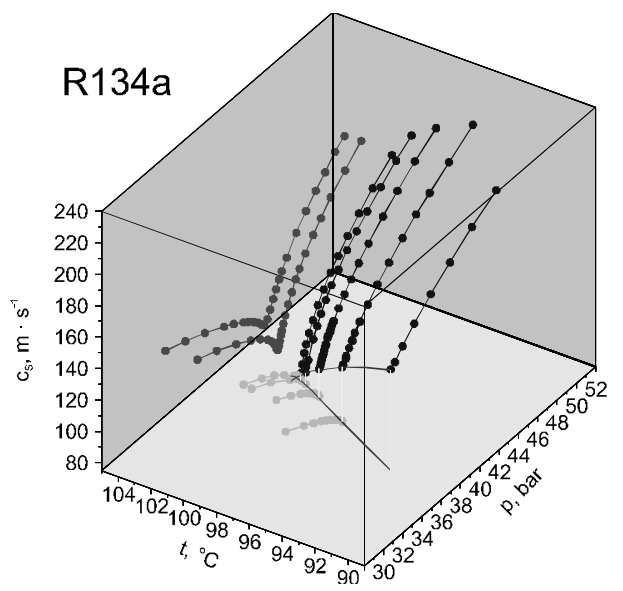

Figure 11 Sound velocity of R134a in a wide range around the critical point [44]

typically only a few hundreds of nanoseconds, even if small scattering angles are employed, which requires the use of fast correlators. The other problem is of a more fundamental nature and is caused by a lack of definition of the scattering vector $[3,45]$. Due to the finite width of stops in the detection optics there is always some angular spread, which is a combination of a simple geometrical and a diffraction effect. This uncertainty does not have a significant effect on probing the Rayleigh line, but causes a spread in the position of the Brillouin line. Thus, the linewidth probed in a spectroscopic experiment is a convolution of the distribution of positions and the actual linewidth.

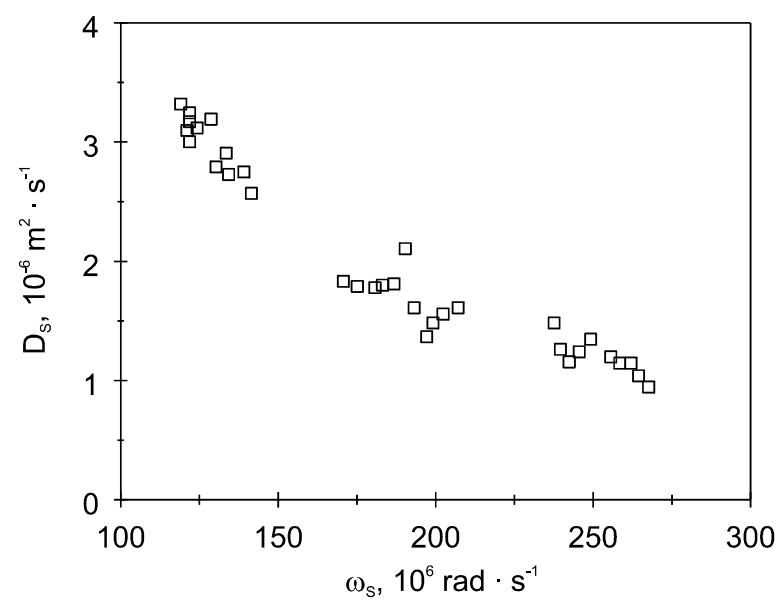

Figure 12 Frequency dispersion with the measurement of sound attenuation; sample: refrigerant $\mathrm{R} 125$, saturated liquid at $45^{\circ} \mathrm{C}$ [45]. 
Analyzing the scattered light in time-domain one obtains in the presence of this effect a decay time shorter than associated with the sound attenuation. However, one has to realize experimental conditions, where the angular uncertainty is as small as possible. This may be done by putting the stops in the detection optics as far as 4-6 m apart from each other.

These difficulties result in the fact that the accuracy of measuring $D_{\mathrm{S}}$ is limited to about $10 \%$, which still is a good deal better than by direct spectroscopic methods. Finally, it must be pointed out that all measurements of sound velocity and attenuation are basically dependent on the actual frequency used in the experiment. This dispersion effect has turned out to be negligible for many fluids and the frequencies probed by DLS as the sound velocity is considered, but is marked for the sound attenuation, especially of refrigerants, cf. Fig. 12.

\subsection{Landau-Placzek Ratio}

The Landau-Placzek ratio $S$ is defined as the ratio of intensities of the Rayleigh $\left(I_{\mathrm{R}}\right)$ to Brillouin $\left(I_{\mathrm{B}}\right)$ lines and is related to the specific heats $c_{\mathrm{p}}$ and $c_{\mathrm{v}}$ by $S=I_{\mathrm{R}} /\left(2 I_{\mathrm{B}}\right)=$ $\left(c_{\mathrm{p}}-c_{\mathrm{v}}\right) / c_{\mathrm{v}}$. The Landau-Placzek ratio may be obtained from the evaluation of the contrasts in various heterodyne experiments, when a local oscillator with defined intensity is added [46].

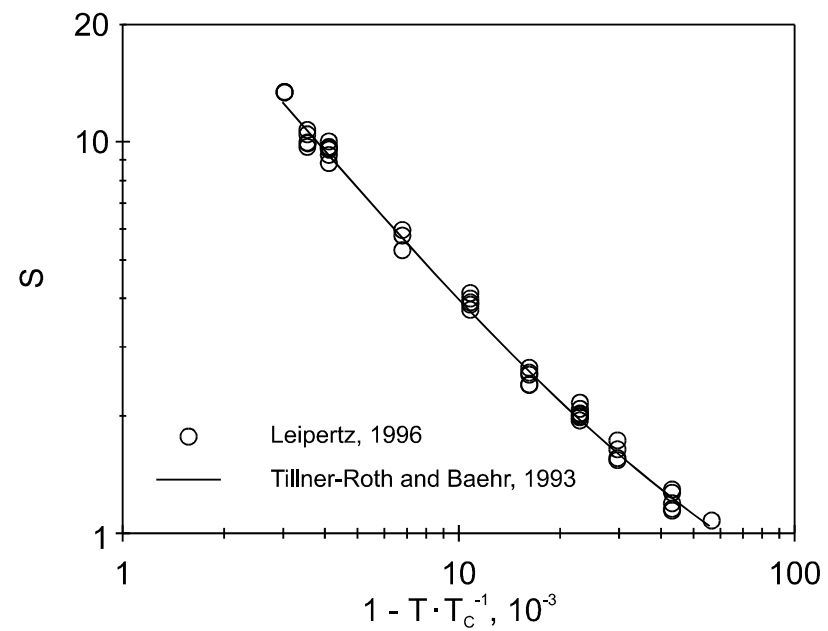

Figure 13 Landau-Placzek ratio for refrigerant R134a obtained by DLS measurements [47] in comparison with reference data from the equation of state [48].

\subsection{Dynamic Viscosity}

The determination of the dynamic viscosity $\eta$ of liquids is in a respect different from the measurement of the other properties treated so far, as it requires the addition of seed particles. The quantity, which is actually measured, is the translational particle diffusion coefficent $D_{\mathrm{P}}$ of spherical particles. Thus, two possible fields of applications open here. 
One area of research is the determination of particle size distributions and, as macromolecules may be regarded as special particles, the determination of molecular weight $[49,50]$. Here, the other application related with particle diffusion coefficients shall be given some more consideration, namely the determination of the dynamic viscosity [51-53].

Generally, as $D_{\mathrm{P}}$ is orders of magnitude smaller than the other diffusion coefficients in the Rayleigh-Brillouin spectrum, this quantity is normally probed at large scattering angles. Another difference is that for the determination of the viscosity low-power lasers such as He-Ne or diode lasers suffice in most cases, as the scattering cross section of the particles is usually much larger than that of other fluctuations in the fluid itself. Accordingly, a homodyne detection scheme is normally employed, and the measurements are not affected by other components in the spectrum.

A key to a successful determination of the viscosity is the choice of suitable particles in a size range of some $20 \mathrm{~nm}$ to $500 \mathrm{~nm}$. These are to be spherical and monodisperse, or at least are to exhibit a narrow size distribution, in order to ensure a correlation function which matches the model of a pure exponential, which is essential for a reliable data evaluation. Moreover, they must be chemically stable and form stable dispersions. For these reasons it is essential to use low particle volume fractions of typically $10^{-4}$ and to vary the particle concentration and/or scattering angle.

An experimental result is shown in Fig. 14, where the viscosity of n-heptane was measured to check the technique. This liquid was chosen, because there are reliable reference data [54].

As the numerical value of the mutual diffusion coefficient is in between those of the thermal diffusivity and the particle diffusion coefficient, prospects open of a simultaneous measurement of $\eta$ and $\mathrm{D}_{12}$ [ 55].

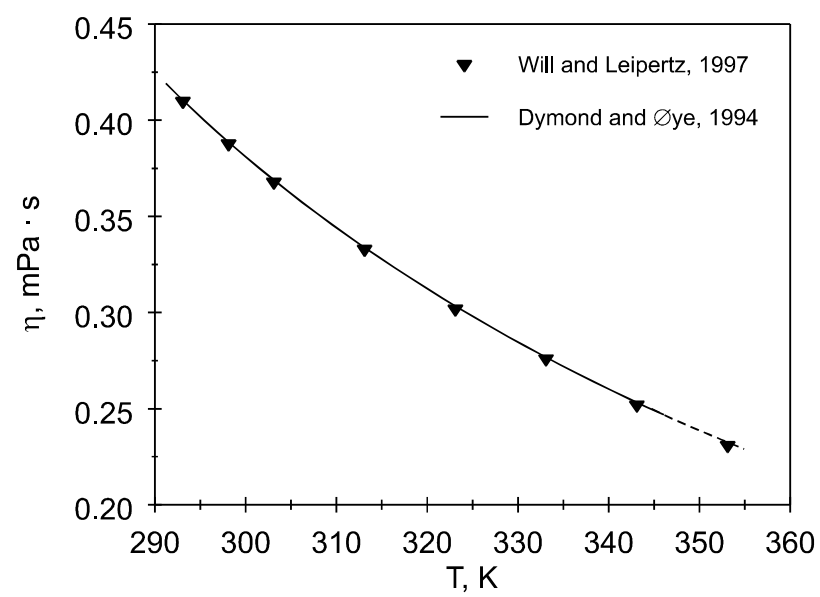

Figure 14 Viscosity of liquid n-heptane probed by dynamic light scattering [53] in comparison to reference data [54]. 


\subsection{Viscosity and Surface Tension}

Although it has been shown that SLS may be performed in complete analogy to DLS measurements from bulk fluids using an identical set-up [56, 57], some differing design features are often employed for SLS measurements. One obvious difference is that SLS normally investigates interfaces in horizontal orientation within a sample cell with light impinging from above. Commonly, scattered light is observed near the reflected beam [58], i.e. also in the top direction, which eases the optical access and is of course essential for non-transparent fluids. Alternatively, for transparent fluids scattered light may be observed close to the direction of the refracted beam [13, 14, 20, 59], which is advantageous due to stability considerations and scattering intensities. SLS experiments typically use small scattering angles of about $0.1^{\circ}$ up to a few degrees. The according range of scattering vectors reaches from an order of $10^{4} \mathrm{~m}^{-1}$ to about $10^{6} \mathrm{~m}^{-1}$, where large $q$-vectors can be achieved easier with a transmission geometry due to intensity considerations. An advantage of larger scattering vectors is that spectral broadening effects due to an uncertainty $\delta q$ in the scattering vector do not play a role in the higher $q$-range.

As in many applications of DLS also the variant surface light scattering makes it possible to determine two properties simultaneously, in the case of SLS this measurement is performed without any extra effort and with surface tension and viscosity yields two quantities whose determination by other means requires two completely different sets of experimental equipment. While commonly the kinematic viscosity $v$ is regarded as the property directly accessible in SLS experiments, it should be stressed that this is only true with the simplifying neglect of gas phase properties. With a proper execution of the method, however, no measurable differences between the values obtained by SLS and conventional methods, in part relying on bulk properties, could be found [12-14]. This is

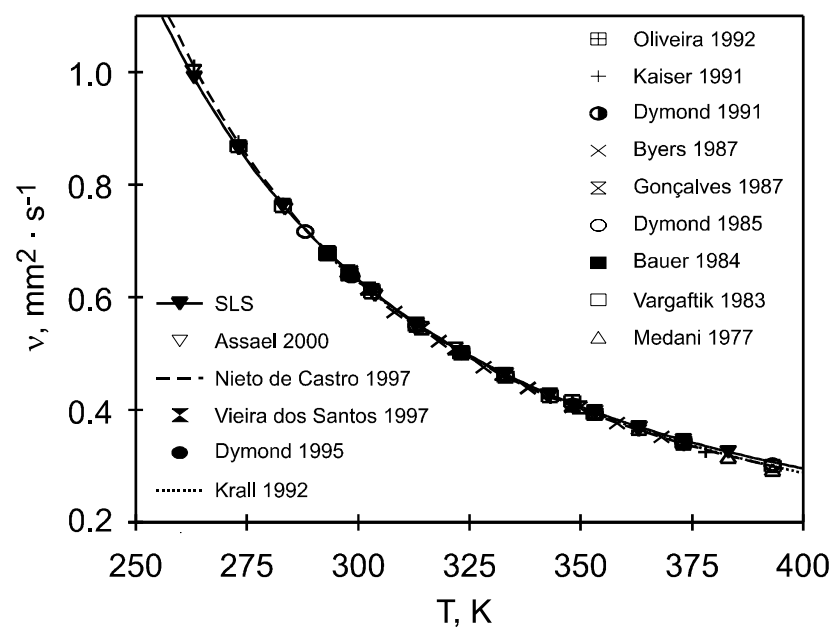

Figure 15 Liquid kinematic viscosity of toluene under saturation conditions from SLS [14] in comparison with literature data [60-73]. 


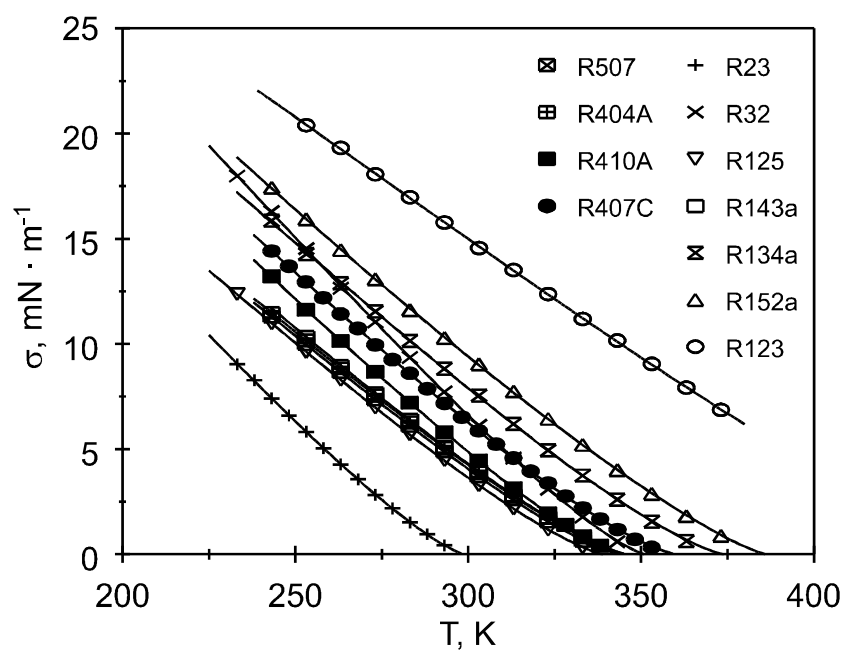

Figure 16 Surface tension of alternative refrigerants and their mixtures under saturation conditions from surface light scattering SLS [75].

pointed up in Fig. 15, where data from SLS [14] for the liquid kinematic viscosity of the important, and, thus well documented reference fluid toluene under saturation conditions are shown in comparison with available literature data from the last twenty years [60-73].

An accuracy of down to $1 \%$ for either property can be obtained with SLS [12-14, 74]. This value is at least comparable with the uncertainty of conventional methods, and together with the unanimous advantage of a simultaneous determination of surface tension and viscosity makes the method interesting also for a routine measurement of these properties of simple fluids and mixtures [12, 20, 37, 38]. An example for measurements on various new pure refrigerants and their mixtures is given in Fig. 16 [75].

Within the scope of the measurement of transport and other thermophysical properties of fluids, high-viscosity fluids have also been studied using the SLS technique. For highviscosity fluids where an overdamped behavior of surface fluctuations is observed, apart from the limiting case close to the critical damping, the quantity which can be directly evaluated from the SLS experiment is the ratio of the dynamic viscosity to the surface tension. Thus, the derivation of the dynamic viscosity requires reliable information about the surface tension. In Fig. 17 the viscosity of diisodecylphthalate (DIDP) resulting from a combination of data from SLS [76] and the pendant drop method [77] is compared with data obtained by other techniques [77-79]. The results for DIDP, which has been proposed by the International Association of Transport Properties (IAPT) as a new viscosity standard for high-viscosity fluids, demonstrate the applicability of the SLS technique to fluids covering a wide range of viscosity, from about $10 \mathrm{mPas}$ to 1 Pas with an inaccuracy of smaller than $1 \%$. Yet, a value of 1 Pas seems not to be the upper limit of the viscosity range that could be investigated by the SLS technique. An estimation 


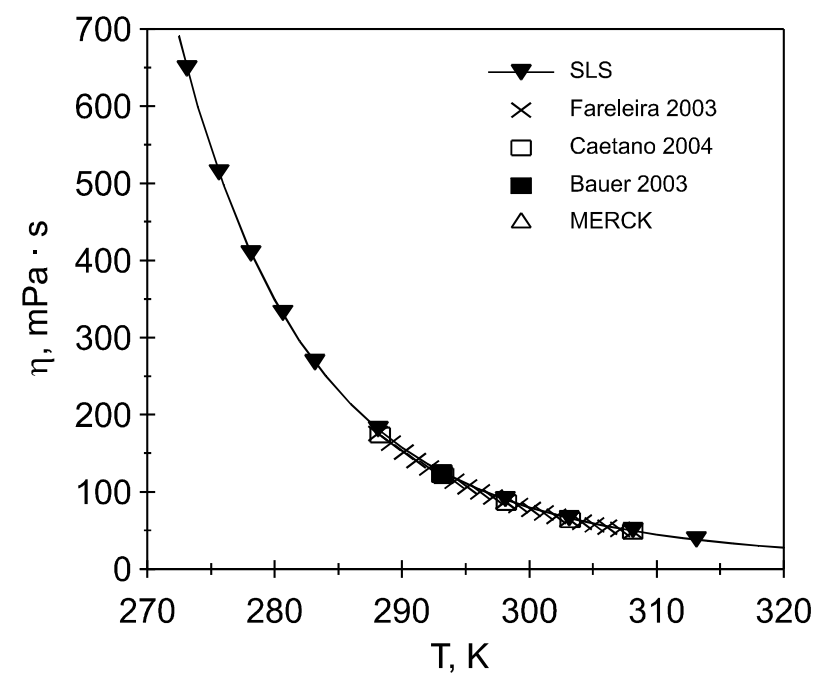

Figure 17 Dynamic viscosity of liquid DIDP under saturation conditions from SLS [76] in comparison with literature data [77-79].

based on the time domain between a few nanoseconds to several hundred milliseconds where PCS can be applied in a sensible way indicates for the upper limit of the viscosity a value of the order of magnitude of $1 \mathrm{kPas}$.

Also, apart from studying specific surface properties the contactless operation makes SLS ideally suited for the investigation of high-temperature melts [80], yet presently with clear limitations in accuracy.

\section{Conclusion}

Light scattering techniques have been widely employed for the investigation of diffusive processes and the determination of transport and other thermophysical properties. The reasons for the success of these methods may be summarized as follows. Measurements may be performed in or close to thermodynamic equilibrium, almost without an input of energy. Experiments are conducted in a non-contact mode, allowing access to regions of thermodynamic state which can hardly be probed by other techniques. The methods allow the determination of a wide range of transport and other thermophysical properties, in some instances even simultaneously. The techniques are based on simple and rigid working equations, where the reliability of the measurements can easily be checked. As the state of development is different for the various properties major tasks for future work will be both the routine application of light scattering to data acquisition for interesting samples over a wide range of temperature and pressure and the continuous improvement of the experimental techniques themselves. Specific challenges are further applications to samples like high-temperature melts and the simultaneous determination of several properties within a single experiment. 


\section{References}

[1] E. Gulari, R. J. Brown, C. J. Pings, AIChE J. 19, 1196 (1973)

[2] M. Corti, V. Digorgio, J. Phys. C 8, 953 (1975)

[3] G. Simonsohn, Opt. Acta 30, 875, 1675 (1983)

[4] K. J. Czworniak, H. C. Anderson, R. Pecora, Chem. Phys. 11, 451 (1975)

[5] L. Onsager, Phys. Rev. 37, 405 (1931); 38, 2265 (1931)

[6] B. J. Berne, R. Pecora, Dynamic Light Scattering with Applications to Chemistry, Biology, and Physics, Robert E. Krieger, Malabar 1990

[7] B. Chu, Laser Light Scattering: Basic Principle and Practice, Academic, Boston 1991

[8] D. Langevin, Light Scattering by Liquid Surfaces and Complementary Techniques, Marcel Dekker, New York 1992

[9] A. Leipertz, A. P. Fröba, in Diffusion in Condensed Matter - Methods, Materials, Models, eds. P. Heitjans, J. Kärger, Springer, Heidelberg 2005, pp. 571-611.

[10] L. D. Landau, E. M. Lifschitz, Lehrbuch der theoretischen Physik, Bd. 6, Hydrodynamik, Akademie, Berlin 1991

[11] A. Einstein, Z. f. Elektroch. 17, 235 (1908)

[12] A. P. Fröba, Dr.-Ing. thesis, Friedrich-Alexander-Universität Erlangen-Nürnberg 2001

[13] A. P. Fröba, A. Leipertz, Int. J. Thermophys. 22, 41 (2001)

[14] A. P. Fröba, A. Leipertz, Int. J. Thermophys. 24, 895 (2003)

[15] W. Brouwer, R. K. Pathria, Phys. Rev. 163, 200 (1967)

[16] M. v. Smoluchowski, Ann. d. Phys. 25, 205 (1908)

[17] R. H. Katyl, U. Ingard, Phys. Rev. Lett. 19, 64 (1967)

[18] R. H. Katyl, U. Ingard, Phys. Rev. Lett. 20, 248 (1968)

[19] S. Hård, Y. Hamnerius, O. Nilsson, J. Appl. Phys. 47, 2433 (1976)

[20] A. P. Fröba, S. Will, A. Leipertz, Int. J. Thermophys. 21, 1225 (2000)

[21] J. Meunier, D. Cruchon, M. A. Bouchiat, C. R. Acad. Sci. (Paris) 268, 422 (1969)

[22] A. D. May, M. Hubert, V. Ghaem-Maghami, Can. J. Phys. 56, 1168 (1978)

[23] V. Ghaem-Maghami, A. D. May, Phys. Rev. A 22 692; 22698 (1980)

[24] D. S. Chung, K. Y. Lee, E. Mazur, Appl. Phys. B 64, 1 (1997)

[25] M. A. Bouchiat, J. Meunier, J. de Phys. (Paris) 32, 561 (1971)

[26] W. H. Press, Numerical Recipes in C, Cambridge University, Cambridge 1992

[27] D. E. Koppel, J. Chem. Phys. 57, 4814 (1974)

[28] S. Will, A. Leipertz, Appl. Opt. 32, 3813 (1993)

[29] A. Leipertz, Int. J. Thermophys. 9, 897 (1988)

[30] P. Jany, J. Straub, Int. J. Thermophys. 8, 165 (1987)

[31] M. Hendrix, A. Leipertz, M. Fiebig, G. Simonsohn, Int. J. Heat Mass Transfer 30, 333 (1987)

[32] K. Kraft, M. Matos Lopes, A. Leipertz, Int. J. Thermophys. 16, 423 (1995)

[33] M. Ibreighith, M. Fiebig, A. Leipertz, G. Wu, Fluid Phase Equil. 80, 323 (1992) 
[34] K. Kraft, A. Leipertz, Proc. CFCs, The Day After (Padua, Italy, 1994), pp. 435442

[35] K. Kraft, A. Leipertz, Fluid Phase Equil. 125, 245 (1996)

[36] A. P. Fröba, S. Will, A. Leipertz, Int. J. Thermophys. 22, 1021 (2001)

[37] A. P. Fröba, S. Will, A. Leipertz, Int. J. Thermophys. 22, 1349 (2001)

[38] A. P. Fröba, A. Leipertz, Int. J. Thermophys. 24, 1185 (2003)

[39] A. P. Fröba, H. Kremer, A. Leipertz, Int. J. Thermophys. 25, 1115 (2004)

[40] G. Wu, M. Fiebig, A. Leipertz, Int. J. Heat and Mass Transfer 31, 1471 (1988)

[41] A. P. Fröba, S. Will, A. Leipertz, Int. J. Thermophys. 21, 603 (2000)

[42] G. Wu, M. Fiebig, A. Leipertz, Wärme- Stoffübertr. 22, 365 (1988)

[43] K. Kraft, A. Leipertz, Appl. Opt. 32, 3886 (1993)

[44] K. Kraft, A. Leipertz, DKV-Tagungsberichte 22 II.1, 199 (1995)

[45] K. Kraft, A. Leipertz, Int. J. Thermophys. 16, 445 (1995)

[46] B. Hinz, G. Simonsohn, M. Hendrix, G. Wu, A. Leipertz, J. Mod. Opt. 34, 1093 (1987)

[47] A. Leipertz, Fluid Phase Equil. 125, 219 (1996)

[48] R. Tillner-Roth, H. D. Baehr, J. Phys. Chem. Ref. Data 23, 657 (1994)

[49] B. Dahneke (ed.), Measurement of Suspended Particles by Quasi-Elastic Light Scattering, Wiley, New York 1984

[50] W. Brown (ed.), Dynamic Light Scattering: The Method and Some Applications, Oxford Science, London 1993

[51] D. F. Williams, C.H. Byers, J. Chem. Eng. Data 32, 2534 (1987)

[52] S. Will, A. Leipertz, Int. J. Thermophys. 16, 433 (1995)

[53] S. Will, A. Leipertz, Int. J. Thermophys. 18, 1339 (1997)

[54] J. H. Dymond, H. A. Øye, J. Phys. Chem. Ref. Data 23, 41 (1994)

[55] S. Will, A. Leipertz, Int. J. Thermophys. 20, 791 (2000)

[56] A. P. Fröba, S. Will, A. Leipertz, Appl. Opt. 36, 7615 (1997)

[57] A. P. Fröba, S. Will, A. Leipertz, Fluid Phase Equil. 161, 337 (1999)

[58] T. Nishio, Y. Nagasaka, Int. J. Thermophys. 16, 1087 (1995)

[59] T. M. JØrgensen, Meas. Sci. Technol. 3, 588 (1992)

[60] C. A. Nieto de Castro, F. J. Vieira dos Santos, private communication, Department of Chemistry and Biochemistry, University of Lisbon (1997)

[61] M. S. Medani, M. A. Hasan, Can. J. Chem. Eng. 55, 203 (1977)

[62] J. H. Dymond, M. A. Awan, N. F. Glen, J. D. Isdale, Int. J. Thermophys. 12, 275 (1991)

[63] J. H. Dymond, N. F. Glen, J. D. Isdale, M. Pyda, Int. J. Thermophys. 16, 877 (1995)

[64] J. H. Dymond, J. Robertson, Int. J. Thermophys. 6, 21 (1985)

[65] H. Bauer, G. Meerlender, Rheol. Acta 23, 514 (1984)

[66] C. H. Byers, D. F. Williams, J. Chem. Eng. Data 32, 344 (1987)

[67] F. A. Gonçalves, K. Hamano, J. V. Sengers, J. Kestin, Int. J. Thermophys. 8, 641 (1987)

[68] B. Kaiser, A. Laesecke, M. Stelbrink, Int. J. Thermophys. 12, 289 (1991) 
[69] N. B. Vargaftik, Tables on the Thermophysical Properties of Liquids and Gases in Normal and Dissociated States, Hemisphere, Washington D.C., 1983

[70] A. H. Krall, J. V. Sengers, J. Kestin, J. Chem. Eng. Data 37, 349 (1992)

[71] C. M. B. P. Oliveira, W. A. Wakeham, Int. J. Thermophys. 21, 773 (1992)

[72] F. J. Vieira dos Santos, C. A. Nieto de Castro, Int. J. Thermophys. 18, 367 (1997)

[73] M. J. Assael, N. K. Dalaouti, J. H. Dymond, Int. J. Thermophys. 21, 291 (2000)

[74] A. P. Fröba, L. Penedo Pellegrino, A. Leipertz, Int. J. Thermophys. 25, 1323 (2004)

[75] A. P. Fröba, A. Leipertz, DKV-Tagungsberichte 29 II.1, 155 (2002)

[76] A. P. Fröba, A. Leipertz, Int. J. Thermophys., submitted 2005

[77] J. M. N. A. Fareleira, private communication, Department of Chemistry and Biochemistry, University of Lisbon (2003)

[78] H. Bauer, private communication, Physikalisch-Technische Bundesanstalt (PTP), Braunschweig (2003)

[79] F. J. P. Caento, J. M. N. A. Fareleira, C. M. B. P. Oliveira, W. A. Wakeham, Int. J. Thermophys. 25, 1311 (2004)

[80] M. Ohnishi, Y. Nagasaka, High Temp.-High Press. 32, 103 (2000) 